\title{
BRICK DECOMPOSITIONS AND $Q$-MANIFOLDS
}

\author{
D. W. CURTIS AND G. KOZLOWSKI
}

\begin{abstract}
A brick decomposition (respectively, generalized brick decomposition) of a metric space $Y$ is a locally finite, star-finite closed cover $\left\{Y_{\alpha}\right\}$ such that each nonempty intersection $Y_{\alpha_{1}} \cap \cdots \cap Y_{\alpha_{n}}, n>1$, is a compact AR (respectively, locally compact AR). Let $K$ be the nerve of the decomposition $\left\{Y_{\alpha}\right\}$, let $Q$ be the Hilbert cube, and $Q_{0}=Q \backslash$ point $\approx Q \times$ $[0,1)$. Then $Y \times Q \approx|K| \times Q$ (respectively, $Y \times Q_{0} \approx|K| \times Q_{0}$ ).
\end{abstract}

1. Introduction. Borsuk [1] showed that if a finite-dimensional compactum admits a brick decomposition (a finite cover by compact AR's whose nonempty intersections are also AR's), then it has the homotopy type of the nerve of the decomposition. Holsztynski [6] later removed the finite-dimensional hypothesis. In this paper we use recent techniques and results of infinite-dimensional topology to show that a compactum $Y$ has the simple homotopy type of the nerve of its brick decomposition $\left\{Y_{i}\right\}$, in the sense that the $Q$-manifold $Y \times Q$ is homeomorphic to the $Q$-manifold nerve $\left\{Y_{i}\right\} \times Q$ ( $Q$ is the Hilbert cube). This holds more generally for locally compact spaces and their locally finite decompositions.

A previous result in this direction appeared in [3], where it was shown that if $X$ and $Y$ admit order-isomorphic $Q$-factor decompositions satisfying appropriate $Z$-set conditions, then $X \times Q$ and $Y \times Q$ are homeomorphic. This theorem was initially used in the proof of a CE-mapping theorem for the PL category, with subsequent applications being made in the theory of hyperspaces ([5], [7]). The decomposition theorem of the present paper is an improvement on this earlier result, in that the $Z$-set hypotheses are dropped, and the conclusion is stated directly in terms of the nerve of a decomposition. At the same time, we obtain corresponding results for generalized brick decompositions by locally compact AR's. Some of these results (specifically, Corollary 2 below) have been motivated by decompositions of certain growth hyperspaces [4].

2. Statement of results. All spaces considered are locally compact separable metric. Let $Q_{0}=Q \times[0,1)$. The one-point compactification $Q_{0} \cup \infty$ is the cone over $Q$, which is homeomorphic to $Q$, and thus $Q_{0}$ is homeomorphic to $Q \backslash$ point.

Definition. A $Q$-decomposition (respectively, $Q_{0}$-decomposition) of a space

Received by the editors May 30, 1977 and, in revised form, December 12, 1977.

AMS (MOS) subject classifications (1970). Primary 54F40, 57A20; Secondary 57C10.

Key words and phrases. Brick decomposition, nerve of a cover, simple homotopy type, $Q$-manifold. 
$Y$ is a locally finite, star-finite closed cover $\left\{Y_{\alpha}\right\}$ such that each nonempty intersection $Y_{\alpha_{1}} \cap \cdots \cap Y_{\alpha_{n}}, n \geqslant 1$, is a copy of $Q$ (respectively, $Q_{0}$ ).

THEOREM. Let $\left\{Y_{\alpha}\right\}$ be a $Q$-decomposition of $Y$, and let $K$ be the nerve of $\left\{Y_{\alpha}\right\}$. Then $Y \times Q \approx|K| \times Q$. Similarly, if $\left\{Y_{\alpha}\right\}$ is a $Q_{0}$-decomposition of $Y$, with nerve $K$, then $Y \times Q \approx|K| \times Q_{0}$.

DEFINITION. A brick decomposition (respectively, generalized brick decomposition) of a space $Y$ is a locally finite, star-finite closed cover $\left\{Y_{\alpha}\right\}$ such that each nonempty intersection $Y_{\alpha_{1}} \cap \cdots \cap Y_{\alpha_{n}}, n \geqslant 1$, is a compact AR (respectively, locally compact AR).

COROLlaRY 1. Let $\left\{Y_{\alpha}\right\}$ be a brick decomposition of $Y$, with nerve $K$. Then $Y \times Q \approx|K| \times Q$. Similarly, if $\left\{Y_{\alpha}\right\}$ is a generalized brick decomposition of $Y$, with nerve $K$, then $Y \times Q_{0} \approx|K| \times Q_{0}$.

Definition. A pointed $Q$-decomposition of a pointed compactum $(Y, p)$ is a finite cover $\left\{Y_{i}\right\}$ such that each $Y_{i}$ contains $p$ and each nontrivial intersection $Y_{i_{1}} \cap \cdots \cap Y_{i_{n}}, n \geqslant 1$, is a copy of $Q$. The relative nerve $K$ of the pointed decomposition is the nerve of the cover $\left\{Y_{i} \backslash p\right\}$ of $Y \backslash p$.

COROLlaRY 2. Let $\left\{Y_{i}\right\}$ be a pointed $Q$-decomposition of $(Y, p)$, with relative nerve $K$, and suppose that $Y \backslash p$ is a $Q$-manifold. Then $Y \approx \operatorname{cone}(|K| \times Q)$.

3. Lemmas and proofs. If $K$ is the nerve of a $Q$-decomposition $\left\{Y_{\alpha}\right\}$, we may consider that the decomposition elements are indexed by the vertices of $K$. Extending this notation, for each simplex $\sigma$ of $K$ we let $Y_{\sigma}$ denote the corresponding nonempty intersection of decomposition elements. We say that $\left\{Y_{\alpha}\right\}$ is a strong $Q$-decomposition if for each pair of simplexes $\sigma, \tau \in K$ with $\sigma<\tau\left(\sigma\right.$ a proper face of $\tau$ ), the intersection $Y_{\tau}$ is a $Z$-set in $Y_{\sigma}$. A strong $Q_{0}$-decomposition is defined similarly. The proof of the Theorem is accomplished by the following lemmas.

LEMMA 1. Let $\left\{Y_{\alpha}\right\}$ be a $Q$-decomposition (respectively, $Q_{0}$-decomposition) of $Y$. Then $Y \times Q$ admits a strong $Q$-decomposition (respectively, strong $Q_{0^{-}}$ decomposition) with nerve isomorphic to the nerve of $\left\{Y_{\alpha}\right\}$.

Lemma 2. Let $\left\{Y_{\alpha}\right\}$ be a strong $Q$-decomposition (respectively, strong $Q_{0^{-}}$ decomposition) of $Y$, with nerve $K$. Then $Y$ is homeomorphic to $|K| \times Q$ (respectively, $|K| \times Q_{0}$ ).

In the proof of Lemma 1 we use Chapman's CE-mapping theorem [2], and West's sum theorem for $Q$-factors [8]. A map $f: X \rightarrow Y$ is a CE-map if it is a proper surjection and each point-inverse $f^{-1}(y)$ has trivial shape. The CEmapping theorem states that if $f: X \rightarrow Y$ is a CE-map, and $X \times Q$ and $Y \times Q$ are $Q$-manifolds, then $X \times Q$ is homeomorphic to $Y \times Q$ and $f \times$ id: $X \times Q \rightarrow Y \times Q$ is a near-homeomorphism. The sum theorem for $Q$-factors states that if $X=X_{1} \cup X_{2}$ is a union of $Q$-factors whose inter- 
section $X_{1} \cap X_{2}$ is also a $Q$-factor, then $X$ is a $Q$-factor.

In the proof of Lemma 2 we use Anderson's $Z$-set homogeneity theorem for $Q$ [2], and its analogue for $Q_{0}$. The homogeneity theorem states that a homeomorphism between $Z$-sets in $Q$ can be extended to a homeomorphism of $Q$ onto itself. The $Q_{0}$-version is obtained by considering the one-point compactification $Q_{0} \cup \infty \approx Q$, and observing that for $A$ a $Z$-set in $Q_{0}$, $A \cup \infty$ is a $Z$-set in $Q_{0} \cup \infty$.

Proof of Lemma 1. Consider $Q=\Pi_{1}^{\infty} I_{i}$, with each $I_{i}=[0,1]$. We construct a space $\tilde{Y}$ such that $Y \times(0, \ldots) \subset \tilde{Y} \subset Y \times Q$, the projection $\pi$ : $\tilde{Y} \rightarrow Y \times(0, \ldots)$ is a CE-map, and $\tilde{Y}$ admits a strong $Q$-decomposition (respectively, strong $Q_{0}$-decomposition) $\left\{\tilde{Y}_{\alpha}\right\}$ indexed by the vertices of $K$, such that $Y_{\alpha} \times(0, \ldots) \subset \tilde{Y}_{\alpha} \subset Y_{\alpha} \times Q$ for each $\alpha$. Thus $K=\operatorname{nerve}\left\{\tilde{Y}_{\alpha}\right\}$.

Let $\sigma \rightarrow I_{\sigma}$ be a 1-1 correspondence between the simplexes of $K$ and a subcollection of the interval factors $\left\{I_{i}\right\}$ of $Q$. For each simplex $\sigma$ of $K$ let $\tilde{Y}_{\sigma}=Y_{\sigma} \times \Pi\left\{I_{\tau} \mid \tau \geqslant \sigma\right\} \times(0, \ldots) \subset Y_{\sigma} \times Q$, and let $\tilde{Y}=\bigcup\left\{\tilde{Y}_{\sigma} \mid \sigma \in K\right\}$. Each $\tilde{Y}_{\sigma}$ is homeomorphic to $Q$ (respectively, $Q_{0}$ ), and $\tilde{Y}_{\tau}$ is a $Z$-set in $\tilde{Y}_{\sigma}$ if $\sigma<\tau$. Since $\cap\left\{\tilde{Y}_{\alpha} \mid \alpha\right.$ is a vertex of $\left.\sigma\right\}=\tilde{Y}_{\sigma},\left\{\tilde{Y}_{\alpha} \mid \alpha\right.$ is a vertex of $\left.K\right\}$ is a strong $Q$-decomposition (respectively, strong $Q_{0}$-decomposition) of $\tilde{Y}$ with nerve $K$.

An easy induction using the sum theorem for $Q$-factors shows that if $\left\{Y_{\alpha}\right\}$ is a $Q$-decomposition of $Y$, then for each $y \in Y, \cup\left\{Y_{\alpha} \mid y \in Y_{\alpha}\right\}$ is a $Q$-factor. Similarly, if $\left\{Y_{\alpha}\right\}$ is a $Q_{0}$-decomposition of $Y$, then for each $y \in Y$, $\cup\left\{Y_{\alpha} \cup \infty \mid y \in Y_{\alpha}\right\}$ (where " $\cup \infty$ " indicates the one-point compactification) is a $Q$-factor, hence $\cup\left\{Y_{\alpha} \times Q \mid y \in Y_{\alpha}\right\}$ is a $Q$-manifold. Therefore, both $Y \times Q$ and $\tilde{Y} \times Q$ are $Q$-manifolds. Since $\pi: \tilde{Y} \rightarrow Y \times(0, \ldots)$ is clearly a CE-map, there is a homeomorphism $\tilde{Y} \times Q \approx Y \times Q$ by which the decomposition $\left\{\tilde{Y}_{\alpha} \times Q\right\}$ is transported to the desired decomposition of $Y \times Q$.

Proof of LemMa 2. A homeomorphism $h: Y \rightarrow|K| \times Q$ (respectively, $h$ : $\left.Y \rightarrow|K| \times Q_{0}\right)$ is built up inductively, using the dual cell structure of $K$. Let sd $K$ be the standard barycentric subdivision. For each $\sigma \in K$ we consider the dual cell $D(\sigma)=\bigcap\{\operatorname{St}(\alpha ; \operatorname{sd} K) \mid \alpha$ is a vertex of $\sigma\}$. Then $|K|=$ $\cup\{D(\sigma) \mid \sigma \in K\}=\cup\{D(\alpha) \mid \alpha$ is a vertex of $K\}$, each $D(\sigma)$ is a convex cell, and $D(\tau)$ is a $Z$-set in $D(\sigma)$ whenever $\sigma<\tau$. Thus $\{D(\alpha) \times Q\}$ is a strong $Q$-decomposition of $|K| \times Q$, and $\left\{D(\alpha) \times Q_{0}\right\}$ is a strong $Q_{0}$-decomposition of $|K| \times Q_{0}$.

We consider first the case of a strong $Q$-decomposition $\left\{Y_{\alpha}\right\}$. For each maximal simplex $\tau \in K$, choose an arbitrary homeomorphism $h_{\tau}: Y_{\tau} \rightarrow D(\tau)$ $\times Q$. Using the $Z$-set homogeneity theorem, we inductively obtain homeomorphisms $h_{\sigma}: Y_{\sigma} \rightarrow D(\sigma) \times Q$, for each $\sigma \in K$, such that $h_{\sigma \mid Y_{\tau}}=h_{\tau}$ whenever $\sigma<\tau$. The desired homeomorphism $h$ is obtained by setting $h(y)=h_{\alpha}(y)$ for $y \in Y_{\alpha}$. The construction in the case of a strong $Q_{0^{-}}$ decomposition is identical, using the $Z$-set homogeneity theorem for $Q_{0}$. 
REMARK. Lemma 2 (in the case of a $Q$-decomposition) is in fact an application of the decomposition theorem of [3], since the strong $Q$ decomposition $\left\{Y_{\alpha}\right\}$ and $\{D(\alpha) \times Q\}$ are order-isomorphic in the sense of [3]. Note that the homeomorphism $h: Y \rightarrow|K| \times Q$ constructed above satisfies $h\left(Y_{\alpha}\right)=D(\alpha) \times Q=\operatorname{St}(\alpha$; sd $K) \times Q$ for each vertex $\alpha$ of $K$. Since the near-homeomorphism $\pi \times$ id: $\tilde{Y} \times Q \rightarrow Y \times Q$ constructed in the proof of Lemma 1 takes each $\tilde{Y}_{\alpha} \times Q$ onto $Y_{\alpha} \times Q$, it is easily seen that for $\left\{Y_{\alpha}\right\}$ a $Q$-decomposition with nerve $K$, there exists a homeomorphism $g: Y \times Q \rightarrow$ $|K| \times Q$ such that $g\left(Y_{\alpha} \times Q\right) \subset \operatorname{St}(\operatorname{St}(\alpha ; K)$; sd $K)$ for each $\alpha$. Similarly for a $Q_{0}$-decomposition.

Proof OF COROLlaRY 1. Using Edwards' product theorem [2] (every locally compact metric ANR is a $Q$-manifold factor), and characterization theorems of Chapman [2] (every compact contractible $Q$-manifold is homeomorphic to $Q$, and every contractible $Q$-manifold which is $[0,1)$-stable is homeomorphic to $Q_{0}$ ), we see that $\left\{Y_{\alpha} \times Q\right\}$ is a $Q$-decomposition of $Y \times Q$ (respectively, $\left\{Y_{\alpha} \times Q_{0}\right\}$ is a $Q_{0}$-decomposition of $\left.Y \times Q_{0}\right)$. Thus $Y \times Q \approx(Y \times Q) \times$ $Q \approx|K| \times Q$ (respectively, $\left.Y \times Q_{0} \approx\left(Y \times Q_{0}\right) \times Q \approx|K| \times Q_{0}\right)$.

An alternative proof for the compact case of Corollary 1 , which is independent of the Theorem (and therefore gives another proof of the Theorem in the case of $Q$-decompositions), is also based on Edwards' product theorem and Chapman's CE-mapping theorem, but uses an intermediary space. Let $W=\cup\left\{Y_{\sigma} \times|\sigma| \mid \sigma \in K\right\} \subset Y \times|K|$, and let $f: W \rightarrow Y$ and $g: \mathrm{W} \rightarrow|K|$ be the projection maps. One verifies that $W$ is an ANR and that $f$ and $g$ are CE-maps, and concludes that $f \times$ id: $W \times Q \rightarrow Y \times Q$ and $g \times$ id: $W \times Q$ $\rightarrow|K| \times Q$ are near-homeomorphisms.

Proof of Corollary 2. This follows from stability for the $Q$-manifold $Y \backslash p$, and the fact that $\left\{Y_{\alpha} \backslash p\right\}$ is a $Q_{0}$-decomposition of $Y \backslash p$ with nerve $K$. Thus $Y \backslash p \approx(Y \backslash p) \times Q \approx|K| \times Q_{0}$. Forming the one-point compactification yields $Y \approx(Y \backslash p) \cup \infty \approx(|K| \times Q \times[0,1)) \cup \infty=\operatorname{cone}(|K| \times$ Q).

Some interesting applications of Corollary 2 have been made to growth hyperspaces [4].

\section{REFERENCES}

1. K. Borsuk, On the imbedding of systems of compacta in simplicial complexes, Fund. Math. 35 (1948), 217-234.

2. T. A. Chapman, Lectures on Hilbert cube manifolds, CBMS Regional Conf. Ser. in Math., no. 28, Amer. Math. Soc., Providence, R. I., 1976.

3. D. W. Curtis, Simplicial maps which stabilize to near-homeomorphisms, Compositio Math. 25 (1972), 117-122.

4. 271-283.

, Growth hyperspaces of Peano continua, Trans. Amer. Math. Soc. 238 (1978),

5. D. W. Curtis and R. M. Schori, Hyperspaces which characterize simple homotopy type, General Topology and Appl. 6 (1976), 153-165.

6. W. Holsztynski, On spaces with regular decompositions, Bull. Acad. Polon. Sci. 12 (1964), 607-611. 
7. R. M. Schori and J. E. West, The hyperspace of the closed unit interval is a Hilbert cube, Trans. Amer. Math. Soc. 213 (1975), 217-235.

8. J. E. West, Sums of Hilbert cube factors, Pacific J. Math. 54 (1974), 293-303.

Departmint of Mathematics, louisiana State Universtty, Baton Rouge, louisiana 70803

Department of Mathematics, Auburn University, Auburn, Alabama 36830 